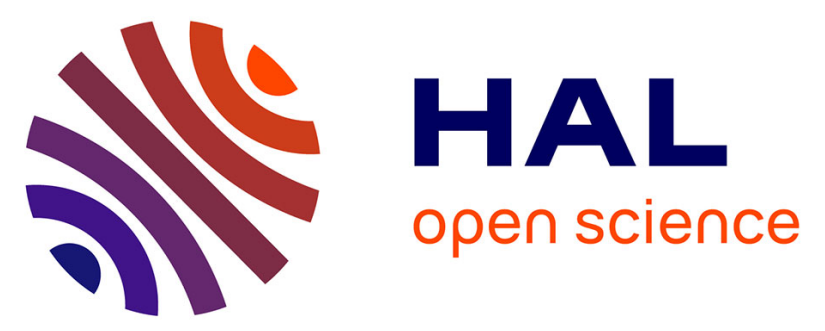

\title{
Moderate oral supplementation with docosahexaenoic acid improves platelet function and oxidative stress in type 2 diabetic patients
}

Evelyne Véricel, Romain Colas, Catherine Calzada, Quang Huy Lê, Nathalie Feugier, Christine Cugnet, Hubert Vidal, Martine Laville, Philippe Moulin, Michel Lagarde

\section{To cite this version:}

Evelyne Véricel, Romain Colas, Catherine Calzada, Quang Huy Lê, Nathalie Feugier, et al.. Moderate oral supplementation with docosahexaenoic acid improves platelet function and oxidative stress in type 2 diabetic patients: DHA amends platelet function and redox status. Thrombosis and Haemostasis, 2015, 114 (1), [Epub ahead of print]. 10.1160/TH14-12-1003 . inserm-01140217

\section{HAL Id: inserm-01140217 https://www.hal.inserm.fr/inserm-01140217}

Submitted on 8 Apr 2015

HAL is a multi-disciplinary open access archive for the deposit and dissemination of scientific research documents, whether they are published or not. The documents may come from teaching and research institutions in France or abroad, or from public or private research centers.
L'archive ouverte pluridisciplinaire HAL, est destinée au dépôt et à la diffusion de documents scientifiques de niveau recherche, publiés ou non, émanant des établissements d'enseignement et de recherche français ou étrangers, des laboratoires publics ou privés. 


\title{
Moderate oral supplementation with docosahexaenoic acid improves platelet function and oxidative stress in type 2 diabetic patients
}

\author{
Evelyne Véricel $^{1}$; Romain Colas ${ }^{1}$; Catherine Calzada ${ }^{1}$; Quang Huy Lêê ${ }^{1}$; Nathalie \\ Feugier $^{2}$; Christine Cugnet ${ }^{3}$; Hubert Vidal ${ }^{1}$; Martine Laville ${ }^{1,2}$; Philippe Moulin ${ }^{1,3}$; \\ Michel Lagarde ${ }^{1}$ \\ ${ }^{1}$ Université de Lyon, Inserm UMR 1060, Laboratoire CarMeN, Université Claude Bernard \\ Lyon 1, INSA-Lyon, Villeurbanne, France; ${ }^{2}$ Centre de Recherche en Nutrition Humaine \\ Rhône-Alpes, Pierre-Bénite, France; ${ }^{3}$ Hôpital Cardiovasculaire Louis Pradel, Lyon-Bron, \\ France
}

Corresponding author:

Evelyne Véricel

UMR Inserm U.1060 / Université de Lyon / INSA-Lyon,

Cardiovasculaire, Métabolisme, Diabétologie et Nutrition CarMeN,

IMBL, Bât Louis Pasteur, INSA, 20 Ave A. Einstein

69621 Villeurbanne Cedex, France

Tel : $33(0) 472438479$

Fax : $33(0) 472438524$

E-mail : evelyne.vericel@insa-lyon.fr

Running title: DHA amends platelet function and redox status

Financial support:

This work was supported by Inserm and a grant from the Fondation Cœur \& Artères. 


\section{Summary}

Platelets from patients with type 2 diabetes are characterized by hyperactivation and high level of oxidative stress. Docosahexaenoic acid (DHA) may have beneficial effects on platelet reactivity and redox status. We investigated whether moderate DHA supplementation, given as a triglyceride form, may correct platelet dysfunction and redox imbalance in patients with type 2 diabetes. We conducted a randomized, double-blind, placebo-controlled, two-period crossover trial $(n=11$ post-menopausal women with type 2 diabetes) to test the effects of $400 \mathrm{mg} /$ day of DHA intake for 2 weeks on platelet aggregation, markers of arachidonic acid metabolism, lipid peroxidation status, and lipid composition. Each 2 week-period was separated from the other by a 6-week washout. Daily moderate dose DHA supplementation resulted in reduced platelet aggregation induced by collagen $(-46.5 \%$, $\mathrm{p}<0.001)$, and decreased platelet thromboxane $\mathrm{B}_{2}(-35 \%, \mathrm{p}<0.001)$, urinary 11-dehydrothromboxane $\mathrm{B}_{2}(-13.2 \%, \mathrm{p}<0.001)$ and $\mathrm{F} 2$-isoprostane levels $(-19.6 \%, \mathrm{p}<0.001)$ associated with a significant increase of plasma and platelet vitamin E concentrations $(+20 \%$ and $+11.8 \%$, respectively, $\mathrm{p}<0.001$ ). The proportions of DHA increased both in plasma lipids and in platelet phospholipids. After placebo treatment, there was no effect on any parameters tested. Our findings support a significant beneficial effect of low intake of DHA on platelet function and a favorable role in reducing oxidative stress associated with diabetes.

Keywords: platelet aggregation; arachidonic acid; vitamin E; isoprostane; plasma and platelet lipid composition 


\section{Introduction}

In 2035, the number of people affected by diabetes mellitus could reach more than 550 million in the world (1). Moreover, cardiovascular disease is one of mortality causes in type 2 diabetes (2). Indeed, it is fully recognized that long-term macrovascular complications are the main cause of morbidity and mortality associated with diabetes. In type 2 diabetes, a complex interplay occurs between hyperglycemia and oxidative stress. Factors that may contribute to increased oxidative stress in diabetes mellitus include protein glycation, increased formation of reactive oxygen species, and antioxidant deficiencies $(3,4)$. On the other hand, it is generally admitted that platelet activation contributes to the increased incidence of thrombotic and atherosclerotic diseases. During platelet activation, several steps occur including the release of arachidonic acid (ARA) from membrane phospholipids. Once released, ARA can be oxygenated into thromboxane $\mathrm{A}_{2}\left(\mathrm{TxA}_{2}\right)$, a potent pro-aggregatory and vaso-constricting compound (5) that rapidly breaks down to form the stable and inactive product thromboxane $\mathrm{B}_{2}\left(\mathrm{TxB}_{2}\right)$. Moreover, we have shown that increased platelet aggregation is already detectable in diabetic patients who do not suffer from vascular complications linked with impairment of antioxidant mechanisms (6).

For several decades, there has been accumulating evidence that n-3 polyunsaturated fatty acids (PUFA) could protect against cardiovascular diseases and interestingly, there is a low prevalence of diabetes and coronary heart disease in populations known for high intake of n-3 fatty acids $(7,8)$. Because of the pivotal role of platelets in the regulation of hemostasis and athero-thrombosis, some studies have focused on the effects of n-3 PUFA on these cells. It is widely accepted that eicosapentaenoic (EPA or 20:5n-3) and docosahexaenoic (DHA or 22:6n-3) acids can decrease platelet function (9). Moreover, DHA could be associated with reduced progression of coronary atherosclerosis (10). Despite these described protective 
effects, some studies failed to report a beneficial association between n-3 PUFA consumption and cardiovascular events (11). Among some explanations for these discordant results, some pertain to the high unsaturation of these PUFA. Indeed, DHA is highly oxidisable owing to the presence of 6 double bonds, and it is known that lipid peroxidation provides some peroxidised lipids increasing the oxidative stress. Such a lipid peroxidation has been implicated to the pathogenesis of cardiovascular diseases (12). Previously, we have shown a bimodal in vitro effect of DHA with antioxidant and pro-oxidant effects at low and high concentrations, respectively (13). More recently, we have conducted an ex-vivo study, with healthy volunteers, which has shown the validity of our in vitro findings (14). Following ingestion of increasing doses of DHA $(200,400,800,1600 \mathrm{mg} /$ day of DHA for 2 weeks each), we have shown that platelet reactivity was decreased after 400 and $800 \mathrm{mg}$ DHA/day. It was concluded that low consumption of DHA (400 to $800 \mathrm{mg} /$ day) could be an effective "treatment" to protect healthy volunteers from platelet-related cardiovascular events.

These data prompted us to perform a randomized double-blind study to determine whether a modest intake of DHA (400 mg/day for 2 weeks) may be also beneficial in type 2 diabetic patients, a population characterized by platelet hyperactivity, impaired antioxidant defense, and increased oxidative stress, in opposing that oxidative stress or synergizing with it.

\section{Materials and methods}

\section{Materials}

All chemicals were purchased from Sigma-Aldrich (Saint-Quentin, Fallavier, France). All solvents were provided by Carlo Erba-Reactifs (Val de Reuil, France). Silica gels 60 plates were supplied by Merck (VWR International, Strasbourg, France). 


\section{Ethics statement}

Informed, written consents were obtained from all participants and the protocol was approved by the local ethic committee, the "Comité de Protection des Personnes Sud-Est II" (ClinicalTrials.gov Identifier is NCT01150292).

\section{Study design}

Eleven post-menopausal women with type 2 diabetes participated. Subjects with diabetes had recent glycated hemoglobin (HbA1c: $7.89 \pm 1.27 \%, 62.8 \pm 14.1 \mathrm{mmol} / \mathrm{mol}, \mathrm{n}=11)$, antidiabetic drugs but no drugs known to affect platelet function (acetylsalicylic acid, clopidogrel, gliclazide, ticlopidine, non-steroidal anti-inflammatory drugs). A randomized, placebo-controlled clinical trial was conducted. The subjects were randomly assigned in double-blind fashion to DHA or placebo, administered for 2 weeks. After baseline visit, subjects were randomly assigned to take 2 capsules/day of DHA or placebo with meals for 2 weeks, then, after 6 weeks of wash out, subjects took 2 capsules/day of placebo or DHA (Figure 1). Capsules of DHA (Pro-Mind) were supplied by Decola (Maldegem, Belgium). Each capsule contained $200 \mathrm{mg}$ DHA, as the only PUFA, in triglycerides from algal oil, 175 mg gelatin, $0.125 \mathrm{mg}$ DL-alpha-tocopherol, and $0.125 \mathrm{mg}$ ascorbic palmitate. Placebo capsules were made, specifically upon our request, by Ayanda GmbH \& Co.KG (Pritwalk, Germany) and contained the same quantities of antioxidants and triglycerides of sunflower oil. Placebo and DHA capsules were indistinguishable and independently packaged by the Central Pharmacy of the hospital (Hospices Civils de Lyon). Pharmacists dispensed placebo or DHA capsules in a random process. All study personnel and participants were blinded to treatment assignment and the code was only revealed to the researchers after laboratory analyses were completed. A dietary record was obtained on the first visit and dietary changes monitored at each visit. 
Blood and urine samples were collected after overnight fasting before and after each supplementation. Urine samples were stored immediately at $-80^{\circ} \mathrm{C}$ in presence of butylated hydroxy-toluene (BHT).

\section{Plasma lipid measurements}

Total cholesterol, HDL-, LDL- cholesterol and triglyceride concentrations were measured using commercial enzymatic kits. Intra-assay and inter-assay coefficients of variation were $<10 \%$.

\section{Plasma preparation and isolation of platelets}

Blood samples were collected in tubes containing acid-citrate-dextrose $(0.8 \%$ citric acid, $2.2 \%$ sodium citrate, and $2.45 \%$ dextrose, $6: 1 \mathrm{v} / \mathrm{v}$ ) and platelets were isolated as described previously (15). The supernatant removed from above the platelet pellet was centrifuged for $10 \mathrm{~min}$ at $18^{\circ} \mathrm{C}$ to obtain platelet-poor plasma.

\section{Platelet aggregation}

Collagen (Horm, from Nycomed, Linz, Austria) was used as agonist to induce platelet aggregation in a Chronolog dual-channel aggregometer (Coulter, Margency, France) according to the turbidimetric method of Born (16). Intra-assay and inter-assay coefficients of variation were $5 \%$ and $6 \%$, respectively, with a lower detection limit of $<0.1 \%$.

\section{Arachidonic acid metabolism}

Metabolism of exogenous arachidonic acid

The oxygenation of ARA through the lipoxygenase and cyclooxygenase pathways was determined by incubating 2.2 microM of $\left[1-{ }^{14} \mathrm{C}\right]$ ARA (specific activity $2.07 \mathrm{GBq} / \mathrm{mmol}$, G.E. Healthcare, Vélisy-Villacoublay) with platelets for $4 \mathrm{~min}$ at $37^{\circ} \mathrm{C}$. Following lipid 
extraction and thin-layer chromatography (TLC) separation, oxygenated products were visualized and quantified with a TLC analyser radioscanner (Raytest, Paris, France) (17). Intra-assay and inter-assay coefficients of variation were $9 \%$ and $10 \%$, respectively, with a lower detection limit of $<0.1 \%$.

Basal formation of platelet thromboxane $\mathrm{B}_{2}$ and its urinary 11-dehydro metabolite

In the absence of specific stimulation, platelets in presence of BHT as an antioxidant $\left(5 \times 10^{-5}\right.$ mol/l) were immediately frozen. $\mathrm{TxB}_{2}$ was quantified by immunoassay according to the manufacturer's recommendations (Enzo Life Sciences, Lyon, France) (Intra-assay and interassay coefficients of variation were 4\%). Spot urine samples were collected and stored immediately at $-80^{\circ} \mathrm{C}$ in presence of $\mathrm{BHT}$, and then 11 -dehydro- $\mathrm{TxB}_{2}$ was also quantified by immunoassay. Intra-assay and inter-assay coefficients of variation were $12 \%$ and $13 \%$, respectively, and the limit of detection was $10 \mathrm{pg} / \mathrm{ml}$ for both kits.

\section{Antioxidant status}

Plasma and platelet vitamin $\mathrm{E}$

Alpha- and gamma-tocopherol concentrations were determined according to a described method (18). Briefly, after extraction with hexane, tocopherol isomers were separated by reverse-phase HPLC and detected by fluorimetry (excitation and emission at $295 \mathrm{~nm}$ and 340 $\mathrm{nm}$, respectively). Intra-assays and inter-assay coefficients of variation were $<5 \%$ and the limit of detection was 4 pmol.

\section{Lipid peroxidation markers}

The malondialdehyde (MDA) content of unstimulated platelets was measured by the HPLC technique of Therasse and Lemonier (19) with fluorimetric detection (excitation $515 \mathrm{~nm}$, 
emission $553 \mathrm{~nm}$ ). Intra-assay and inter-assay coefficients of variation were $12 \%$ and $15 \%$, respectively, and the limit of detection was 5 pmol.

Urinary isoprostane (8-iso- $\mathrm{PGF}_{2 \text { alpha }}$ or $\left.15-\mathrm{F} 2 \mathrm{t}-\mathrm{IsoP}\right)$ and creatinine were determined by immunoassay (Enzo Life Sciences, Lyon, France) and urinary isoprostane expressed per milligram of creatinine. Intra-assays and inter-assays coefficient of variations were $5 \%$ and $9 \%$, respectively and the limit of detection was $15 \mathrm{pg} / \mathrm{ml}$ for isoprostane kit. Intra-assay and inter-assay coefficients of variation were $4 \%$ and $3 \%$, respectively, and the limit of detection was $0.3 \mu \mathrm{g} / \mathrm{ml}$ for creatinine kit.

\section{Lipid analysis}

Plasma lipids were extracted twice with chloroform/ethanol (2:1, v/v) containing BHT and 1,2-diheptadecanoyl-sn-glycero-3-phosphocholine as an internal standard and transmethylated with methanol (20). Fatty acid methyl esters (FAMEs) were analyzed by gas chromatography (GC) and values were calculated as mole percentages (mol\%) of all FAMEs determined $\left(\mathrm{C}_{16}\right.$ to $\left.\mathrm{C}_{24}\right)$.

Platelet lipids were extracted twice with chloroform/ethanol $(2: 1, \mathrm{v} / \mathrm{v})$ containing BHT and 1,2-diheptadecanoyl-sn-glycero-3-phosphocholine and 1,2-diheptadecanoyl-sn-3phosphoethanolamine as internal standards. Phosphatidylcholine (PC) and phosphatidylethanolamine (PE) were separated by TLC, transmethylated with methanol and analyzed by GC. Intra-assay and inter-assay coefficients of variation were between $3 \%$ and $10 \%$ according to the fatty acid, and the limit of detection was $10 \mathrm{ng}$.

\section{Sample size calculation}

The sample size calculation was based on our previous data. Considering that, first the percent of aggregation was increased in diabetic patients compared to controls (6), and 
second 400 mg-DHA ingestion in healthy volunteers decreased platelet aggregation induced by collagen (14), we estimated that 13 subjects would give $80 \%$ power to detect a $40 \%$ difference in platelet aggregation induced by collagen at a significance level of $\mathrm{P}=0.05$. 14 diabetic patients were recruited but 3 voluntarily withdrew.

\section{Statistical analysis}

The study was designed as a two-period crossover study with double blinding to the order of randomization to DHA or placebo. Statistical analyses were performed by using StatView 5 for Windows (Abacus Corp, Baltimore, MD, USA). The non-parametric Friedman test was used to analyze the differences for all the variables. Wilcoxon's post-test was used to identify group differences. Results are presented as mean \pm SEM $(n=11)$.

\section{Results}

\section{Subjects' characteristics}

Eleven post-menopausal women with type 2 diabetes were included in the protocol analysis. Their mean age was $59.8 \pm 4.7$ years at baseline. Some anthropometric variables were measured such as BMI $\left(34.1 \pm 5.1 \mathrm{~kg} / \mathrm{m}^{2}\right)$, waist and hip circumferences $(113.7 \pm 10.1 \mathrm{~cm}$ and $115 \pm 10.5 \mathrm{~cm}$, respectively, $\mathrm{n}=9$ to 11$)$. Patients followed the DHA and placebo supplementations without any reported difficulty. According to patient self-reports, lifestyle and medication were unchanged throughout the study. As reported in Table 1, there were no significant differences in metabolic parameters measured during placebo or DHA treatment.

\section{Platelet aggregation}


As shown in Figure 2, platelet aggregation induced by $0.05 \mu \mathrm{g} / \mathrm{ml}$ collagen was significantly decreased (-46.5\%) after the $400 \mathrm{mg} / \mathrm{d}$ DHA supplementation compared to baseline. No effect was observed after placebo treatment.

\section{Platelet metabolism}

Incubations of platelets with exogenous ARA allowed us to determine the specific oxygenation of this fatty acid by cyclooxygenase and 12-lipoxygenase. Interestingly, DHA intake affected significantly the formation of $\mathrm{TxB}_{2}$ with a decrease of $35 \%$ (Table 2). Moreover, 12-hydroxy-5,8,10-heptadecatrienoic acid (HHT), the other main cyclooxygenase product in platelets was decreased by $16.4 \%(\mathrm{p}=0.06)$. Altogether, a significant decrease of the cyclooxygenase pathway was noted whereas the formation of 12-HETE, the lipoxygenase end-product, was not affected by DHA supplementation (Table 2). In the absence of specific stimulation, lower amount of basal $\mathrm{TxB}_{2}$ was found after DHA intake compared to before DHA (Table 2). One major urinary metabolite of $\mathrm{TxB}_{2}$ (11-dehydro-TxB $\mathrm{B}_{2}$ ) was also significantly decreased after DHA supplementation (Table 2). No effect was observed after placebo treatment.

\section{Antioxidant status and lipid peroxidation}

To evaluate the antioxidant status, alpha-tocopherol level, an effective lipophilic antioxidant and free radical scavenger, was determined in both plasma and platelets. As shown in Table 2, significant increases of plasma and platelet alpha-tocopherol levels were observed after DHA intake compared to tocopherol levels before supplementation. A tendency to the increase was observed for platelet gamma-tocopherol levels but the difference did not reach statistical significance (pre DHA: $34.1 \pm 1.7 v s$ post DHA: $39.5 \pm 2.8$ pmol/mg prot., $\mathrm{p}=0.09$, $\mathrm{n}=10$ ). To assess the overall lipid peroxide levels in platelets, platelet MDA contents were determined and a tendency to decrease was observed $(\mathrm{p}=0.06)$ (Table 2) after DHA 
supplementation compared to pre DHA. Finally, we measured a urinary marker of oxidative stress, 8-iso-PGF 2 alpha $($ or $15-\mathrm{F} 2 \mathrm{t}-\mathrm{IsoP}$ ). The level of this marker was significantly decreased after DHA intake compared to the control before supplementation of DHA (Table 2). No effect on the antioxidant status and lipid peroxidation was observed after placebo treatment as compared to the situation before placebo.

\section{Fatty acid composition of plasma and platelet lipids}

In plasma, supplementation of the diet with $400 \mathrm{mg}$ DHA/d for 2 weeks, induced a significant increase of DHA $(+40 \%)$ in total lipids (Fig. 3A). This result reflects acceptable compliance for capsule intake. Interestingly, this change was accompanied by a concomitant slight but significant decrease of $22: 5 n-3 \quad(-9.9 \%)$ while $20: 5 n-3$ proportion did not change significantly. No effect on the main n-6 fatty acids (18:2, 20:3 and 20:4) was observed (Fig. 3B). Regarding platelets, a significant increase of DHA in PC (+36\%) was detected (Fig. 3C) as well as in PE (+29\%) (Fig. 3E). As already found in plasma, the 22:5n-3 proportion decreased significantly in PE (-18.7\%) and a tendency to decrease could be observed in PC (10.7\%). No effect on the main n-6 fatty acids (18:2, 20:3 and 20:4) was observed in PC (Fig. 3D) and PE (Fig. 3F) after DHA supplementation. Placebo supplementation had no significant effect either on plasma lipid composition or platelet phospholipids (Fig. 3 A to F). Proportions of saturated fatty acids (and 16:0 and 18:0 dimethyl-acetals for PE, representing plasmalogens), and monounsaturated fatty acids remained constant for all plasma and platelet lipids (results not shown).

\section{Discussion}


Platelet aggregation is a complex process involving multiple receptors and signaling pathways. Collagen-induced platelet shape change and aggregation involves the participation of its receptors, the release of endogenous arachidonic acid and formation of thromboxane $\mathrm{A}_{2}$, and the response to the latter lipid mediator. Indeed, whole reactivity to collagen could not take into account the reactivity to other specific agonists such as ADP and epinephrine. Because we have previously shown that low supplementation of DHA can reduce platelet aggregation in response to collagen, in healthy volunteers (14), the primary objective of this study was to investigate the efficacy of $400 \mathrm{mg}$ DHA daily supplementation for 2 weeks on platelet function in type 2 diabetic patients, who are characterized by platelet hyperactivity (6). One of the main findings of the study is the demonstration that this modest oral dose of DHA was able to reduce platelet aggregation in response to collagen in post-menopausal women with type 2 diabetes mellitus. In such a population, Woodman et al. (21) and Venkatakrishnan et al. (22) already showed that DHA supplementation reduced collageninduced platelet aggregation, but our result is the first one obtained after such a low DHA supplementation. In addition and in agreement with our data on platelet aggregation, we show for the first time that the conversion of exogenous ARA into cyclooxygenase products was decreased after DHA intake while conversion of ARA by the lipoxygenase pathway was not affected as already observed in vitro (23). Moreover, the basal formation of platelet $\mathrm{TxB}_{2}$ was significantly reduced after DHA as well as the concentration of one major urinary metabolite (11-dehydro- $\mathrm{TxB}_{2}$ ), which represents a biomarker of platelet activity (24), and reflects the whole biosynthesis of $\mathrm{TxA}_{2}$ by platelets and extra-platelet sources. Altogether, our results indicate that moderate DHA intake efficiently decreased platelet reactivity in patients with type 2 diabetes mellitus.

Because of the high susceptibility of DHA to oxidative damage, care was taken to ensure that DHA supplementation did not increase lipid peroxidation. Indeed, numerous studies support 
the conclusion that there is an association between diabetes and oxidative stress. It is known that oxidative stress may be an important factor in the pathogenesis of cardio-vascular diseases (3), and also precedes the development of atherosclerosis as already shown in patients affected by type 1 (25) or type 2 diabetes without vascular complications (6). Previously, it was shown that plasma MDA significantly decreased after n-3 PUFA (EPA+DHA) supplement in patients with type 2 diabetes mellitus (26) or did not increase after DHA or EPA intervention (27). Platelet MDA is considered as a global oxidative stress marker (produced by both enzymatic and non-enzymatic oxidation). Interestingly, we observed a tendency, close to significance, to a decrease of platelet MDA after the $400 \mathrm{mg} / \mathrm{d}$ DHA supplement. Accordingly, F2-isoprostanes, products of free radical peroxidation of ARA, released by PLA $_{2}$ activity to the blood stream and finally excreted into urine, provide a reliable measure of in vivo oxidative stress (28). As already shown by Mori et al. (29), we also found a significant decrease of 15-F2t-isoprostane excretion following DHA intake. These results are also in agreement with those of McDonald et al. (30) who found an n-3 PUFA-mediated decrease in platelet isoprostane levels in a group of patients with type 2 diabetes. However, both studies $(29,30)$ dealt with much higher intake of EPA plus DHA. The augmented oxidative stress reported in diabetic patients $(3,6)$ may be the result of higher free radical production but also caused by decreased antioxidant defenses. For a long time, it has been reported that alpha-tocopherol level was decreased in plasma (31) as well as in platelets from patients with diabetes (6). Interestingly, our evaluation of plasma and platelet alpha-tocopherol levels before and after $400 \mathrm{mg} /$ day DHA supplementation confirmed the efficacy of the intervention. Indeed, consistent with decreased lipid peroxidation, plasma and platelet vitamin E levels were significantly increased as already observed in platelets after low intake of EPA (18) or DHA (14). 
Supplementation of patients with $400 \mathrm{mg}$ /day DHA had no effect on the plasma lipid profile with no changes in the concentrations of total cholesterol, cholesterol fractions and triglycerides but it should be noted that the baseline values were already quite in the normality or borderline high. In agreement, several reports have indicated that DHA supplementation reduced plasma triglycerides with doses superior to $2 \mathrm{~g} /$ day (32) and had no significant effects on total cholesterol and lipid subfractions (33).

Baseline plasma DHA proportion is generally low because this fatty acid is consumed in small quantities in dietary lipids and the synthesis from alpha-linolenic and eicosapentaenoic acids is not efficient in humans. Consequently, a low supplementation like $400 \mathrm{mg} / \mathrm{day}$ DHA induced a substantial increase in plasma lipids $v s$ baseline. We have indeed previously reported that a $200 \mathrm{mg} /$ day DHA dose was sufficient to increase DHA proportion in both plasma phospholipids and cholesteryl esters in healthy men (14). As already reported $(14,34,35)$, we found a decreased $22: 5 n-3$ proportion in response to DHA intake. To explain this decrease, Conquer and Holub (36) had proposed a possible competition between DHA and 22:5n-3 to esterification into phospholipids while other modifications of metabolism could not be ruled out.

The biological actions of DHA may probably be due to different mechanisms induced by the fatty acid itself, esterified or not. Indeed, we cannot exclude some effects of DHA on the molecular organization of plasma membrane according to its incorporation into phospholipids and the remodeling of cholesterol-enriched lipid microdomains (37). The effects of such small doses of DHA on the regulation of numerous gene products (38) have not been shown, but they cannot be excluded either. Moreover, since more than ten years, there is a growing interest for the effects of DHA oxygenated metabolites either on oxidative stress (39) or on platelet aggregation (40). Thus, additional mechanisms may mediate the effects observed in 
the current study. Nevertheless, our results reinforce recommendations for a low intake of DHA but the duration of such a supplement remains to be established.

Altogether, in this randomized study, our findings indicate that a modest DHA intake, not only decreases platelet reactivity, but offers an additional therapeutic benefit in reducing redox status in patients with type 2 diabetes mellitus. Our results reinforce the antioxidant potential of low doses of DHA already suggested in healthy subjects (14).

Acknowledgements. The authors are grateful to all patients for their cooperation. The authors thank and express deep gratitude to Drs Stéphanie Lambert-Porcheron and Migueline Nouvel for the patient recruitment. The authors also thank the staff at the Pharmacie Centrale des Hospices Civils de Lyon for the randomization process and the dispatching of capsules, the nurses at the Department of Endocrinology and at the Centre de Recherche en Nutrition Humaine Rhône-Alpes for their expert blood drawing and some lipid analyses.

Duality of Interest. No potential conflicts of interest relevant to this article were reported.

\section{References}

1. Guariguata L, Whiting DR, Hambleton I, et al. Global estimates of diabetes prevalence for 2013 and projections for 2035. Diabetes Res Clin Pract 2014; 103: 137-149.

2. Barr ELM, Zimmet PZ, Welborn TA, et al. Risk of cardiovascular and all-cause mortality in individuals with diabetes mellitus, impaired fasting glucose, and impaired glucose tolerance: the Australian Diabetes, Obesity, and Lifestyle Study (AusDiab). Circulation 2007; 116: $151-157$.

3. Baynes JW, Thorpe SR. Role of oxidative stress in diabetic complications : a new perspective in diabetic complications: a new perspective on an old paradigm. Diabetes 1999; 48: $1-9$.

4. Zatalia SR, Sanusi H. The role of antioxidants in the pathophysiology, complications, and management of diabetes mellitus. Acta Mad Indonses 2013; 45: 141-147. 
5. Hamberg M, Svensson J, Samuelsson B. Thromboxanes : a new group of biologically active compounds derived from prostaglandin endoperoxides. Proc Natl Acad Sci 1975; 72: 2994-2998.

6. Véricel E, Januel C, Carreras M, et al. Diabetic patients without vascular complications display enhanced basal platelet activation and decreased antioxidant status. Diabetes 2004; 53: 1046-1051.

7. Feskens EJ, Virtanen SM, Rasanen L, et al. Dietary factors determining diabetes and impaired glucose tolerance: a 20-year follow-up of the Finnish and Dutch cohorts of the Seven Countries Study. Diabetes Care 1995; 18: 1104-1112.

8. De Caterina R, Madonna R, Bertolotto A, et al. N-3 fatty acids in the treatment of diabetic patients. Diabetes Care 2007; 30: 1012-1026.

9. Gao LG, Cao J, Mao QX, et al. Influence of omega-3 polyunsaturated fatty acidsupplementation on platelet aggregation in humans: a meta-analysis of randomized controlled trials. Atherosclerosis 2013; 226: 328-334.

10. Erkkilä AT, Matthan NR, Herrington DM, et al. Higher plasma docosahexaenoic acid is associated reduced progression of coronary atherosclerosis in women with CAD. J Lipid Res 2006; 47: 2814-2819.

11. Sacks FM, Stone PH, Gibson CM, et al. (HARP Research Group). Controlled trial of fish oil for regression of human coronary atherosclerosis. J Am Coll Cardiol 1995; 25: 14921498.

12. Yagi K. Lipid peroxides and human diseases. Chem Phys lipids 1987; 45: 337-351.

13. Véricel E, Polette A, Bacot S, et al. Pro- and antioxidant activities of docosahexaenoic acid on human blood platelets. J thromb Haemost 2003; 13: 566-572.

14. Guillot N, Caillet E, Laville M, et al. Increasing intakes of the long-chain omega-3 docosahexaenoic acid: effects on platelet functions and redox status in healthy men. FASEB J 2009; 23: 2909-2916.

15. Lagarde M, Bryon PA, Guichardant M, et al. A simple and efficient method for platelet isolation from their plasma. Thromb Res 1980; 17: 581-588.

16. Born GV. Aggregation of blood platelets by adenosine diphosphate and its reversal. Nature 1962; 194: 927-929.

17. Boukhchache D, Lagarde M. Interactions between prostaglandin precursors during their oxygenation by human platelets. Biochim Biophys Acta 1982; 713: 386-392.

18. Croset M, Véricel E, Rigaud M, et al. Functions and tocopherol content of blood platelets from elderly people after low intake of purified eicosapentaenoic acid. Thromb Res 1990; 57: $1-12$. 
19. Therasse J, Lemonnier F. Determination of plasma lipoperoxides by high performance liquid chromatography. J Chromatogr 1987; 413: 237-241.

20. Bowyer DE,Leat WM, Howard AN, et al. The determination of the fatty acid composition of serum lipids separated by thin-layer chromatography; and a comparison with column chromatography. Biochim Biophys Acta 1963; 70: 423-431.

21. Woodman RJ, Mori TA, Burke V, et al. Effects of purified eicosapentaenoic acid on platelet, fibrinolytic and vascular function in hypertensive type 2 diabetic patients. Atherosclerosis 2003; 166: 85-93.

22. Venkatakrishnan P, Anuradha S, Bhattacharjee J, et al. Effects of low dose of omega-3 fatty acids on platelet functions and coagulation profile in Indian patients with type 2 diabetes mellitus with vascular complications : a prospective, preliminary study. J Indian Acad Clin Med 2007; 8: 45-52.

23. Corey EJ, Shih C, Cashman JR. Docosahexaenoic acid is a strong of prostaglandin but not leukotriene biosynthesis. Proc Natl Acad Sci 1983; 80: 3581-3584.

24. Catella F,Healy D, Lawson, et al. 11-dehydro-thromboxane B2: a quantitative index of thromboxane A2 formation in the human circulation. Proc Natl Acad Sci USA 1986; 83: 5861-5865.

25. Davì G, Chiarelli F, Santilli F, et al. Enhanced lipid peroxidation and platelet activation in the early phase of type 1 diabetes mellitus: role of interleukin- 6 and disease duration. Circulation 2003; 107: 3199-3203.

26. Kesavulu MM, Kameswararao B, Apparao Ch, et al. Effect of omega-3 fatty acids on lipid peroxidation and antioxidant enzyme status in type 2 diabetic patients. Diabetes Metab 2002; 28: 20-26.

27. Azizi-Soleiman F, Jazayeri S, Eghtesadi S. Effects of pure eicosapentaenoic and docosahexaenoic acids on oxidative stress, inflammation and body fat mass in patients with type 2 diabetes. Int J Prev Med 2013; 8: 922-928.

28. Praticò D. F(2)-isoprostanes: sensitive and specific non-invasive indices of lipid peroxidation in vivo. Atherosclerosis 1999; 147:1-10.

29. Mori TA, Woodman RJ, Burke V, et al. Effect of eicosapentaenoic acid and docosahexaenoic acid on oxidative stress and inflammatory markers in treated-hypertensive type 2 diabetic subjects. Free Radic Biol Med 2003; 35: 772-781.

30. McDonald DM, O'Kane F, McConville M, et al. Platelet redox balance in diabetic patients with hypertension improved by n-3 fatty acids. Diabetes Care 2013; 36: 998-1005.

31. Nourooz-Zadeh J, Rahimi A, Tajaddini-Sarmadi J, et al. Relationships between plasma measures of oxidative stress and metabolic control in NIDDM. Diabetologia 1997; 40: 647653. 
32. Pirillo A, Catapano AL. Omega-3 polyunsaturated fatty acids in the treatment of atherogenic dyslipidemia. Atheroscler Suppl 2013; 14: 237-242.

33. Hartweg J, Farmer AJ, Perera R, and al. Meta-analysis of the effects of n-3 polyunsaturated fatty acids on lipoproteins and other emerging lipid cardiovascular risk markers in patients with type 2 diabetes. Diabetologia 2007; 50: 1593-1602.

34. von Schacky C, Weber PC. Metabolism and effects on platelet function of the purified eicosapentaenoic and docosahexaenoic acids in humans. J Clin Invest 1985; 76: 2446-2450.

35. Conquer JA, Holub BJ. Supplementation with an algae source of docosahexaenoic acid increases (n-3) fatty acid status and alters selected risk factors for heart disease in vegetarian subjects. J Nutr 1996; 12: 3032-3039.

36. Conquer JA, Holub BJ. Dietary docosahexaenoic acid as a source of eicosapentaenoic acid in vegetarians and omnivores. Lipids 1997; 32: 341-345.

37. Shaikh SR, Kinnun JJ, Leng X, et al. How polyunsaturated fatty acids modify molecular organization in membranes: Insight from NMR studies of model systems. Biochim Biophys Acta 2014 May 9. pii: S0005-2736(14)00155-2. doi.

38. Jump DB, Botolin D, Wang Y, et al. Fatty acid regulation of hepatic gene transcription. J Nutr 2005; 135: 2503-2506.

39. Mukherjee PK1, Marcheselli VL, Serhan CN, et al. Neuroprotectin D1: a docosahexaenoic acid-derived docosatriene protects human retinal pigment epithelial cells from oxidative stress. Proc Natl Acad Sci 2004;101: 8491-8496.

40. Chen P1, Véricel E, Lagarde M, et al. Poxytrins, a class of oxygenated products from polyunsaturated fatty acids, potently inhibit blood platelet aggregation. FASEB J 2011; 25: 382-388.

Figure legends

Figure 1 -Study design.

Figure 2 Influence of DHA or placebo intake on platelet aggregation.

Percentages of aggregation were assessed $4 \mathrm{~min}$ after the addition of $0.05 \mathrm{microg} / \mathrm{ml}$ collagen.

All values are means \pm SEM, $n=11 . * * \mathrm{p}<0.001$ post DHA vs. pre DHA. 
Figure 3-Effect of the DHA or placebo intake on plasma and platelet lipid composition.

A: Effect of the DHA or placebo supplementation on main n-3 fatty acids in plasma. B:

Effect of the DHA or placebo supplementation of DHA on main n-6 fatty acids in plasma. C: Effect of the DHA or placebo supplementation on main n-3 fatty acids in platelet phosphatidylcholine (PC).-D: Effect of the DHA or placebo supplementation of DHA on main $n-6$ fatty acids in platelet PC. E: Effect of the DHA or placebo supplementation on main n-3 fatty acids in platelet phosphatidylethanolamine (PE). F: Effect of the DHA or placebo supplementation on main n-6 fatty acids in platelet PE.

** $\mathrm{p}<0.001$ post DHA vs pre DHA.

Table1-Characteristics of participants before and after DHA or placebo supplementation

\begin{tabular}{|c|c|c|c|c|}
\hline $\begin{array}{c}\text { Weight } \\
(\mathrm{kg})\end{array}$ & Pre DHA & Post DHA & Pre placebo & Post placebo \\
\hline $\begin{array}{c}\text { Diastolic blood } \\
\text { pressure } \\
(\mathrm{mmHg})\end{array}$ & $75.8 \pm 1.5$ & $83.3 \pm 5$ & $83.7 \pm 4.6$ & $83.8 \pm 4.8$ \\
\hline $\begin{array}{c}\text { Systolic blood } \\
\text { pressure } \\
(\mathrm{mmHg})\end{array}$ & $137 \pm 5.9$ & $141.2 \pm 5.2$ & $137.8 \pm 3.7$ & $137.3 \pm 6.3$ \\
\hline $\begin{array}{c}\text { Triglycerides } \\
(\mathrm{mmol} / \mathrm{l})\end{array}$ & $1.67 \pm 0.8$ & $1.51 \pm 0.16$ & $1.75 \pm 0.17$ & $1.91 \pm 0.24$ \\
\hline $\begin{array}{c}\text { Total cholesterol } \\
(\mathrm{mmol} / \mathrm{l})\end{array}$ & $4.37 \pm 0.23$ & $4.47 \pm 0.33$ & $4.59 \pm 0.30$ & $4.30 \pm 0.25$ \\
\hline $\begin{array}{c}\text { HDL cholesterol } \\
(\mathrm{mmol} / \mathrm{l})\end{array}$ & $1.18 \pm 0.06$ & $1.18 \pm 0.08$ & $1.13 \pm 0.07$ & $1.12 \pm 0.07$ \\
\hline $\begin{array}{c}\text { LDL cholesterol } \\
(\mathrm{mmol} / \mathrm{l})\end{array}$ & $2.41 \pm 0.26$ & $2.53 \pm 0.30$ & $2.54 \pm 0.33$ & $2.35 \pm 0.21$ \\
\hline
\end{tabular}

Data are means \pm SEM $(n=11)$. There were no significant differences between pre DHA and post DHA values and between pre placebo and post placebo values. 
Table 2- Effect of DHA and placebo on platelet arachidonic acid metabolism, antioxidant status and lipid peroxidation

\begin{tabular}{|c|c|c|c|c|}
\hline & Pre DHA & Post DHA & Pre placebo & Post placebo \\
\hline $\begin{array}{c}\text { TxB } \\
\text { (nmol/mg prot.) }\end{array}$ & $0.92 \pm 0.2$ & $0.60 \pm 0.16^{* *}$ & $0.68 \pm 0.11$ & $0.55 \pm 0.07$ \\
\hline $\begin{array}{c}\text { HHT } \\
\text { (nmol/mg prot.) }\end{array}$ & $0.55 \pm 0.09$ & $0.46 \pm 0.10$ & $0.47 \pm 0.06$ & $0.46 \pm 0.05$ \\
\hline $\begin{array}{c}\text { TxB } 2+\text { HHT } \\
\text { (nmol/mg prot.) }\end{array}$ & $1.48 \pm 0.28$ & $1.07 \pm 0.26^{*}$ & $1.15 \pm 0.17$ & $1.02 \pm 0.12$ \\
\hline $\begin{array}{c}12-\text { HETE } \\
\text { (nmol/mg prot.) }\end{array}$ & $0.27 \pm 0.08$ & $0.24 \pm 0.07$ & $0.21 \pm 0.02$ & $0.23 \pm 0.02$ \\
\hline $\begin{array}{c}\text { Basal TxB } 2 \\
\text { (pmol/mg prot.) }\end{array}$ & $102.9 \pm 14$ & $70.9 \pm 12.1^{* *}$ & $81.2 \pm 13.6$ & $85.1 \pm 13.3$ \\
\hline $\begin{array}{c}11-\text { dehydro-TxB } 2 \\
\text { (ng/mg creat.) }\end{array}$ & $6.1 \pm 0.5$ & $5.3 \pm 0.4^{* * *}$ & $5.8 \pm 0.6$ & $6.2 \pm 0.8$ \\
\hline $\begin{array}{c}\text { Plasma vitamin E } \\
\text { (nmol/ml) n=10 }\end{array}$ & $8.5 \pm 0.9$ & $10.2 \pm 0.8^{* *}$ & $11.2 \pm 1.1$ & $10.6 \pm 1.2$ \\
\hline $\begin{array}{c}\text { Platelet vitamin E } \\
\text { (pmol/mg prot.) }\end{array}$ & $220.1 \pm 13.5$ & $246.1 \pm 17.7^{* *}$ & $251.1 \pm 11.9$ & $234.6 \pm 12.1$ \\
\hline $\begin{array}{c}\text { MDA } \\
\text { (pmol/mg prot.) }\end{array}$ & $211.9 \pm 36.4$ & $147.2 \pm 29.3$ & $228.2 \pm 48.1$ & $219.1 \pm 37.6$ \\
\hline $\begin{array}{c}15-F 2 t-I s o P \\
\text { (ng/mg creat.) }\end{array}$ & $5.1 \pm 0.9$ & $4.1 \pm 0.8^{* *}$ & $4.1 \pm 0.7$ & $5.2 \pm 1$ \\
\hline
\end{tabular}

Formation of the main ARA metabolites through cyclooxygenase $\left(\mathrm{TxB}_{2}\right.$ and HHT) and lipoxygenase (12-HETE) produced after stimulation with 2.2 microM $\left[{ }^{14} \mathrm{C}\right]$ ARA during 4 min. at $37^{\circ} \mathrm{C}$ (the first four lines). Platelet lipids were extracted and separated by TLC, and metabolites of $\left[{ }^{14} \mathrm{C}\right]$ ARA were quantified by radio-chromatography. Basal formations of $\mathrm{TxB}_{2}$ in unstimulated platelets (in grey) and of one major urinary metabolites of $\mathrm{TxB}_{2}, 11$ dehydro- $\mathrm{TxB}_{2}$, were quantified by immunoassays. Plasma, platelet vitamin E (alphatocopherol) and platelet MDA were extracted and quantified after HPLC separation. Urinary isoprostanes were quantified by immunoassays. There were no significant differences between pre placebo and post placebo values. All values are means $\pm S E M, n=11$. ${ }^{*} p<0.05$, $* * \mathrm{p}<0.001$ compared to values before DHA (pre DHA). 
Extra-table

What is known about this topic?

- Increased platelet function and oxidative stress are observed in patients with type 2 diabetes.

- DHA may exert athero-protective properties under certain conditions.

- DHA may have a bimodal effect with antioxidant and pro-oxidant at low and high concentrations, respectively.

What this paper add?

- Moderate dose of DHA reduced platelet hyper-aggregation of patients with type 2 diabetes.

- In these conditions, DHA reduced oxidative stress associated with type 2 diabetes.

- Our study supports a beneficial effect of low intake of DHA in patients with type 2 diabetes. 
Fig.1

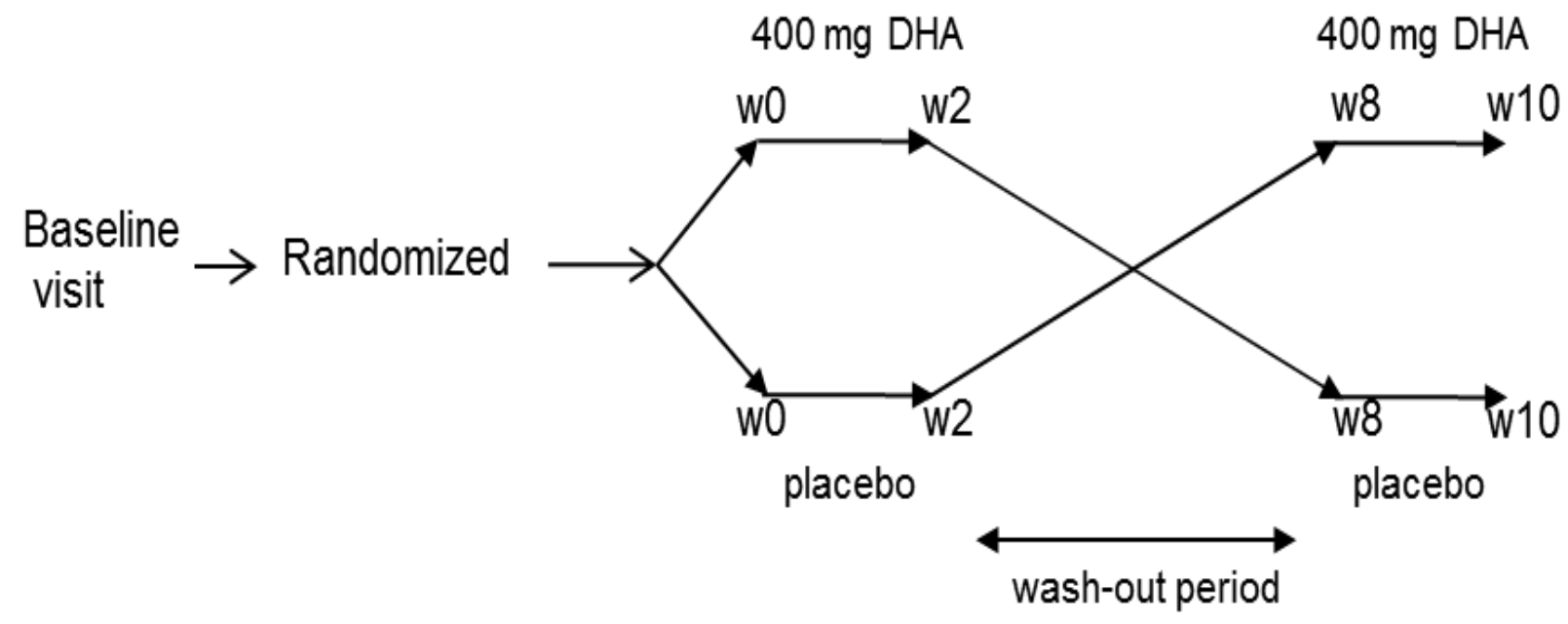


Fig. 2

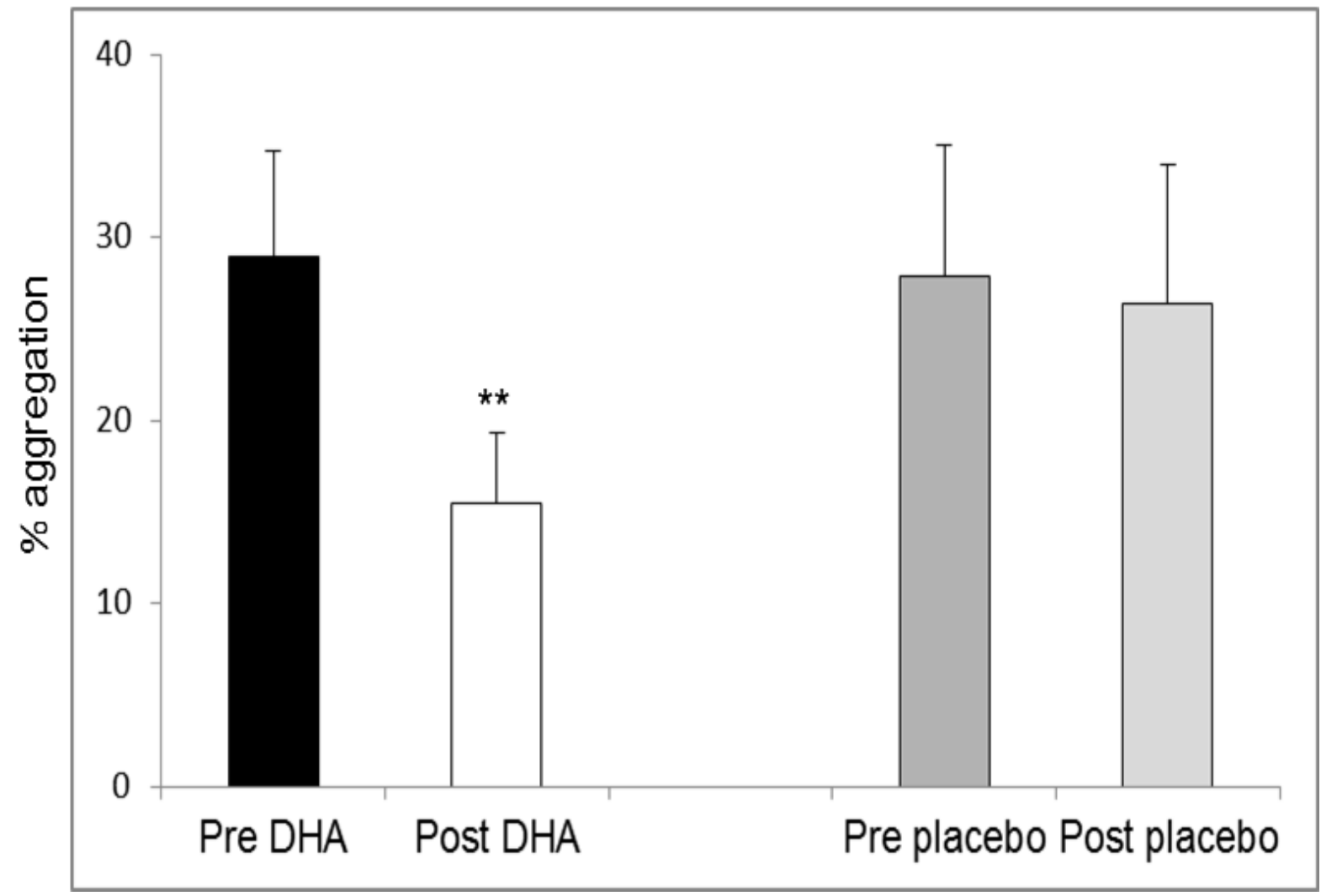


Fig. 3
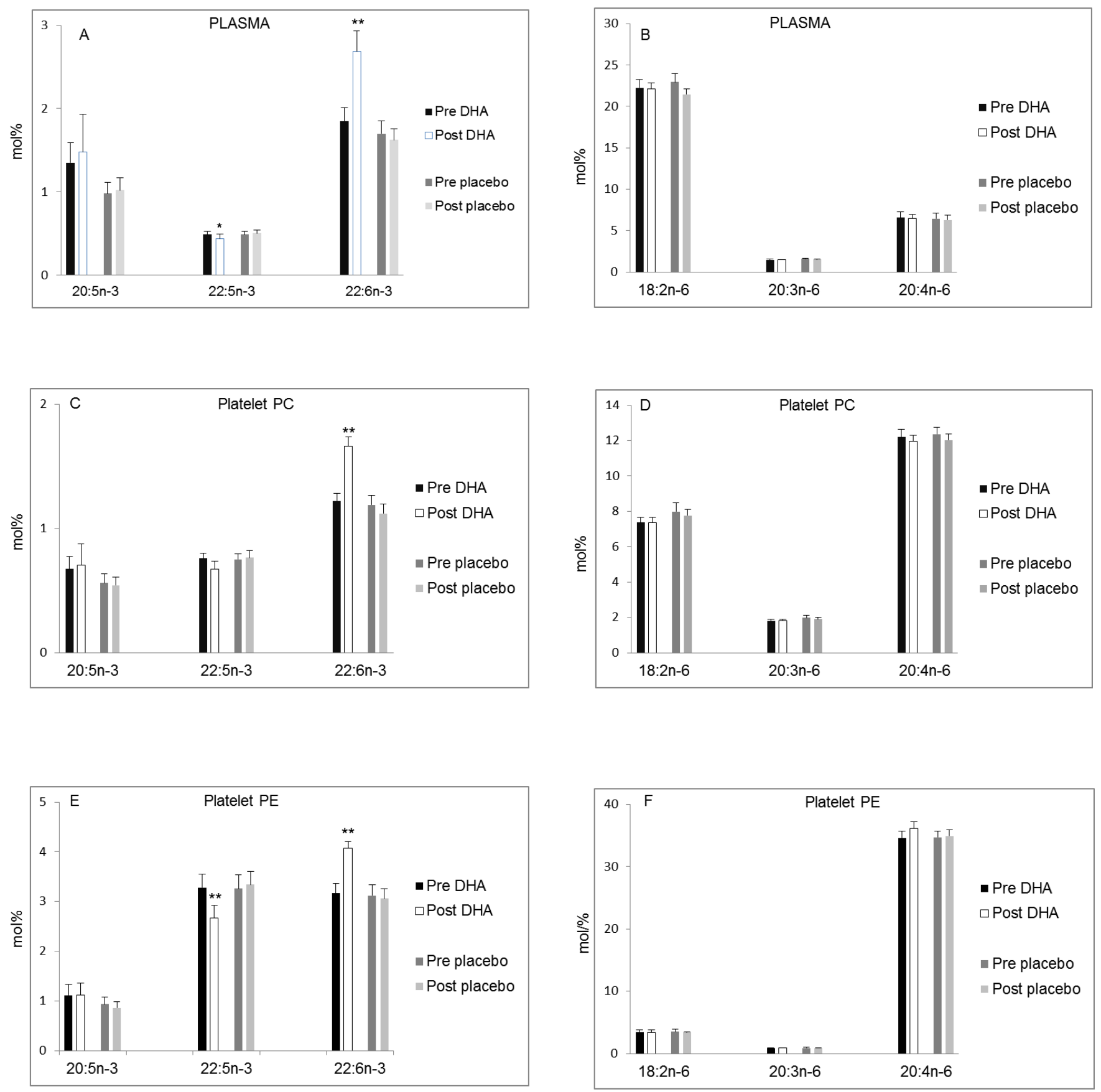